\title{
The American Phytopathological Society \\ Elected and Appointed Officers, Representatives, and Committees for 1998-1999
}

Please note: The most current board and committee rosters are found on-line at APSnet

\section{Council}

President: C. E. Windels

President-Elect: N. K. Van Alfen

Immediate Past President: C. L. Campbell

Vice President: S. A. Slack

Secretary: D. J. Jardine

Treasurer: J. L. Sherwood

Editor-in-Chief, Phytopathology: M. E. Daub

Editor-in-Chief, Plant Disease: G. E. Shaner

Editor-in-Chief, Molecular Plant-Microbe Interactions: J. E. Leach

Editor-in-Chief, Phytopathology News: R. F. Nyvall

Editor-in-Chief, APS Press: S. M. Douglas

Senior Councilor-at-Large: H. R. Dillard

Intermediate Councilor-at-Large: J. E. Loper

Junior Councilor-at-Large: R. C. Gergerich

Councilor of the Caribbean Division: J. E. Polston

Councilor of the North Central Division: P. E. Lipps

Councilor of the Northeastern Division: T. J. Burr

Councilor of the Pacific Division: G. A Chastagner

Councilor of the Potomac Division: E. L. Stromberg

Councilor of the Southern Division: D. T. Beadle

APS Foundation Representative: S. M. Coakley (ex officio)

Executive Vice President: S. C. Nelson (ex officio)

\section{Staff}

Executive Vice President/Publisher: Steven C. Nelson

\section{Representatives}

American Institute of Biological Sciences: M. L. Powelson

American National Standards Institute: J. E. Elson

American Type Culture Collection: J. H. Hill

Coalition for Education in the Life Sciences: E. A. Brown

COFARM: S. A. Tolin

Council for Agricultural Science and Technology: R. W. Smiley, B. J. Jacobsen International Commission on Microbial Ecology: S. Lindow

International Society of Arboriculture: R. J. Stipes

International Society for Plant Pathology: Chair: C. J. Delp. A. Alvarez, J. D.

Froyd, L. V. Madden, C. C. Mundt, L. Sequeira, P. S. Teng, A. H. van Bruggen

International Union of Microbiological Societies: D. C. Gross

National Committee on Worker Safety Standards: S. A. Alexander

National Institutes for the Environment: T. A. Kucharek

National Research Council, Commission on Life Sciences (Corresponding

Society): R. C. Gergerich

National Research Council (Board on Agriculture, Society Representative): C. L. Campbell

Society of Environmental Toxicology and Chemistry: M. Simini

Society of Nematologists: E. L. Davis

APS Foundation, Board of Directors

Chair: S. M. Coakley. Vice Chair: B. J. Jacobsen. Secretary: A. O. Paulus.

Treasurer: A. K. Vidaver. Past Chair: O. W. Barnett. J. S. Batten (ex officio),

M. N. Cline, J. R. French, S. C. Nelson (ex officio), J. L. Sherwood (ex officio)

Office of International Programs Advisory Board

Director: R. A. Bennett. Immediate Past Director: L. Sequeira. G. S. Abawi, L. Black, S. H. De Boer, G. A. Forbes, H. A. Lamey, T. W. Mew, S. C. Nelson (APS

Executive Vice President), R. C. Ploetz, N. W. Schaad, E. B. Smalley, J. R.

Steadman, Z. Xiong, G. A. Zentmyer, APS Foundation representative

Policies and Program(s): Chair: (to be decided). Immediate Past Chair: R. F.

Line. K. S. Cardwell, L. E. Datnoff, C. J. Delp, J. Dubin, J. H. McBeath

National Plant Pathology Board

Chair: A. K. Vidaver. J. Amador, O. W. Barnett, R. J. Cook, D. E. Mathre, L. J. Stowell, S. A. Tolin

Publication no. P-1998-1125-020
Office of Electronic Communication (OEC)

Director: J. D. MacDonald. A. M. Alvarez, C. L. Ash (ex officio), M. N. Cline, S. M. Douglas, D. C. Gross, G. G. Grove, R. C. Ploetz, J. B. Ristaino, K.-B. G. Scholthof, G. L. Schumann, M. Wimer (ex officio), C. E. Windels OEC Electronic Technology Advisory Committee: Chair: C. T. Bull. Vice Chair: D. M. Eastburn. Past Chair: J. B. Ristaino. A. R. Biggs, M. E. Grebus, G. G. Grove, D. B. Marti, D. E. Mayhew, G. W. Moorman, T. D. Murray, K.B. Scholthof, D. W. Thornbury

APS Publications Board

Chair: M. E. Daub. S. M. Douglas, J. E. Leach, J. D. MacDonald, R. F. Nyvall, G. E. Shaner, M. Wimer (ex officio)

OPAE Advisory Board

Director: G. C. Bergstrom. D. A. Albaugh, M. J. Bjerkness (ex officio), J. E. Carroll, M. N. Cline, M. A. Ellis, L. J. Francl, D. C. Gross, M. L. Powelson, A K. Vidaver, C. L. Ash (ex officio), C. L. Campbell (ex officio), A. L. Hope (ex officio), B. D. Mogen, S. C. Nelson (ex officio), J. R. Steadman, C. E. Windels Communications: Chair: D. A. Albaugh. C. L. Ash (ex officio), M. J. Bjerkness (ex officio), R. H. Brlansky, M. N. Cline, R. F. Line, R. F. Nyvall, N. W. Schaad, P. R. Sellers, M. D. Steinberg

Editors of APS PRESS

Editor-in-Chief: S. M. Douglas. Senior Editors: D. M. Benson, A. Bergamin Filho, E. J. Braun, T. J. Burr, C. J. D’Arcy, M. Daughtrey, T. R. Gottwald, R. Loria, R. C. Ploetz, A. H. van Bruggen

Editors of BIOLOGICAL AND CULTURAL TESTS FOR CONTROL OF PLANT DISEASES

Editor: M. T. McGrath. Associate Editor: P. M. Phipps. Section Editors: M. J. Boehm, S. C. Bost, K. L. Bowen, A. R. Chase, D. R. Cooley, T. S. Isakeit, D. J. Jardine, P. E. Lipps, S. A. Miller, G. P. Munkvold

Editors of MOLECULAR PLANT-MICROBE INTERACTIONS

Editor-in-Chief: J. E. Leach. Senior Editors: J. L. Dangl, M. Daniels, S. Lindow, A. Osbourn, P. F. Palukaitis, H. Spaink, V. M. Williamson. Associate Editors: C. Allen, M. Arlat, F. Barras, C. L. Bender, N. J. Brewin, J. P. Carr, D. R. Cook, D. J. Ebbole, F. Garcia-Arenal, J. R. Glazebrook, F. Govers, J. Greenberg, A. M. Hirsch, M. Holsters, H. C. Kistler, W. H. Knogge, J. W. Kronstad, A. J. Maule, K. W. Mendgen, R. S. Nelson, J. E. Parker, S. Perotto, L. S. Pierson III, J. M. Salmeron, F. Sánchez, W. Schaefer, D. Scheel, J. E. Schoelz, F. F. White, N. D. Young

\section{Editors of PHYTOPATHOLOGY}

Editor-in-Chief: M. E. Daub. Senior Editors: M. L. Carson, D. L. Coplin, R. A Dean, M. C. Edwards, B. I. Hillman, H. Leung, C. M. Liddell, J. Mihail, D. M. Weller. Associate Editors: D. Andrivon, N. Benhamou, D. M. Bird, J. K. Brown, D. A. Cuppels, M. J. Davis, K. S. Elias, R. C. French, D. W. Gabriel, S. E. Gold, T. L. Graham, J. Hammond, A. M. Jarosz, Y. Jin, N. J. Keller, R. P. Larkin, M. Mazzola, M. G. Milgroom, C. C. Mundt, R. S. Nelson, S. C. Nelson, C. H. Opperman, B. W. Pennypacker, N. B. Quigley, J. M. Raaijmakers, H. B. Scholthof, D. Shtienberg, L. C. van Loon, M. Wilson, X.-B. Yang

Editors of PLANT DISEASE

Editor-in-Chief: G. E. Shaner. Senior Editors: A. R. Biggs, J. C. Correll, L. J. Francl, D. L. Hopkins, N. R. O’Neill, M. E. Ostry, J. K. Pataky, C. M. Rush, H. D. Shew, H. E. Waterworth. Feature Editor: M. L. Gleason. Spotlight on Diagnosis Editor: C. L. Sutula. Disease Notes Assigning Editor: R. M. Davis. Focus Editor: T. Kommedahl. Associate Editors: H. A. Bolkan, K. L. Bowen, W. C. Chun, B. B. Clarke, P. D. Colyer, D. R. Cooley, J. M. Duniway, J. A. Duthie, K. Eastwell, S. A. Enebak, D. M. Gadoury, R. D. Gitaitis, G. G. Grove, T. C. Harrington, R. M. Hunger, P. J. Landschoot, D. J. MacKenzie, P. S. McManus, S. A. Miller, G. P. Munkvold, W. T. Nishijima, J. P. Noe, J. S. Russin, H. Scherm, D. L. Seifers, J. P. Wilson, C. P. Woloshuk

\section{Society Internal Relations}

Archives and History Awareness: Chair: S. Jensen. Vice Chair: P. D. Peterson. B. J. Christ, R. F. Nyvall, W. Pedersen

Awards and Honors: Chair: N. K. Van Alfen. Vice Chair: J. H. Hill. Immediate Past Chair: D. M. Benson. I. Barash, S. Lindow, D. I. Mills, W. B. Stevenson Committee on Committees: Chair: H. R. Dillard. R. C. Gergerich, J. E. Loper

Executive: Chair: C. L. Campbell. H. R. Dillard, D. J. Jardine, S. C. Nelson, J. L. Sherwood, S. A. Slack, N. K. Van Alfen, C. E. Windels 
Financial Advisory: Chair: J. L. Sherwood. S. M. Douglas, J. Loper, S. C. Nelson (ex officio), S. A. Slack, E. L. Stromberg, N. K. Van Alfen, C. E. Windels (ex officio)

Headquarters Operations: J. L. Sherwood. C. L. Campbell, N. K. Van Alfen, C. E. Windels, AACC representatives

Meeting Site Selection: Chair: J. E. Loper. D. T. Beadle, T. J. Burr, G. A Chastagner, H. R. Dillard, R. C. Gergerich, F. Labatt (ex officio), P. E. Lipps, J. E. Polston, E. L. Stromberg

Nominating: Chair: H. R. Dillard. D. T. Beadle, T. J. Burr, G. A Chastagner, P. E. Lipps, J. E. Polston, E. L. Stromberg

Placement: Chair: R. A. Kuznia. Vice Chair: D. A. Palmer. Past Chair: J. M. Perkins. P. J. Cotty, L. J. du Toit, N. Kolb (ex officio), P. M. McCabe, E. A. Sobek, S. L. Walker

Sustaining Associates: Chair: M. N. Cline. Vice Chair: (to be decided). A. B. Bassi, J. R. French, M. L. Gleason, G. W. Moorman, S. R. Siemer, M. D. Wiglesworth

\section{Society General Policies}

Biotechnology Regulation Impact Assessment: Chair: A. L. Pilgeram. Vice Chair: (to be decided). Past Chair: B. K. Duffy. G. K. Batra, G. J. Defago, M. R. Finkh, J. L. Norelli, D. C. Thompson

Collections and Germ Plasm: Chair: J. A. Thies. Vice Chair: (to be decided). Past Chair: M. L. Carson. L. R. Abad, R. E. Ford, J. A. Foster, J. Hill, S. Leath, R. F. Line, K. McCluskey, M. E. Meadows, M. E. Miller, A. Y. Rossman, J. R. Stavely, C. Thomas, A. T. Tschanz

Cultural Diversity: Chair: (to be decided). Vice Chair: (to be decided). Past Co-Chairs: H. L. Warren and P. L. Hughes. P. D. Adams, A. E. Dorrance, B. K. Duffy, J. E. Lundquist, R. W. Miller, M. A. Morant, N. Ntahimpera, J. J. Roberts, D. K. Villalon

Extension: Chair: P. R. Sellers. Vice Chair: M. A. Ellis. Past Chair: J. P. Damicone. D. M. Eastburn, N. P. Goldberg, D. E. Hershman, S. A. Johnston, R. K. Jones, S. R. Koenning, S. M. Lim, C. A. Smith

Graduate Student: Chair: J. S. Batten. Vice Chair: T. K. Mitchell. Past Chair: W. W. Turechek. M. Anderson, C. A. Bradley, L. R. Emele, T. Glenn, M. Goellner, D. B. Langston, D. Long, C. Riegel, S. M. Tudor, N. Zriba

Industry: Chair: A. J. Julis. Vice Chair: T. Werk. Past Chair: A. J. Julis. B. Carroll, S. R. Foor, B. J. Jacobsen, R. P. Kaiser, T. Lurek, V. Morton, J. L. Riggs, R. Ross, K. K. Shetty, E. C. Tedford

Industry Advisory: Chair: N. B. Carroll. C. M. Becker, M. N. Cline, S. P. Falk, J. R. James, A. J. Julis, R. P. Kaiser, D. F. Kendra, K. T. Kmetz, H. V. Morton, J. L. Riggs, B. J. Shortt, C. L. Sutula

New Fungicide and Nematicide Data: Chair: P. Vincelli. Vice Chair: (to be decided). Past Chair: R. E. Baird. G. T. Berggren, B. A. Fulling, T. L. Kirkpatrick, C. S. Kousik, A. A. MacNab, D. A. Rosenberger, B. S. Sipes, T. B. Sutton, L. W. Timmer, W. F. Wilcox

Phytopathology News Advisory: Chair: R. F. Nyvall. Vice Chair: (to be decided). C. L. Ash (ex officio), J. E. Loper, R. P. Mulrooney, G. L. Schumann, M. Wimer (ex officio)

Private Practice: Chair: J. H. Hart. Vice Chair: (to be decided). Past Chair: D. D. Miller. W. N. Becker, G. Bonn, D. J. Chapman, S. J. Day, C. H. Mellinger, J. J. Muchovej, B. E. Paulsrud, G. W. Simone, M. D. Steinberg

Program: Chair: N. K. Van Alfen. Vice Chair: (to be decided). Past Chair: C. E. Windels. S. C. Nelson, Committe chairs

Regulatory Plant Pathology: Chair: C. L. Schoulties. Vice Chair: (to be decided). Past Chair: S. C. Redlin. K. O. Britton, W. L. Bruckart, J. H. Ghazanfari, E. M. Killgore, S. A. Mengistu, W. Schuh, W. H. Sheta, A. B. Sinderman, X. B. Yang, R. E. Ykema

Teaching: Chair: T. A. Evans. Vice Chair: (to be decided). Past Chair: B. D. Mogen. D. N. Appel, P. Arneson, R. B. Carroll, J. H. Connolly, C. R. Curtis, K. D. Gwinn, M. A. Langham, J. Mullen, M. B. Riley, A. T. Saad, E. L. Stewart, S. Wickes Wescott

Women in Plant Pathology: Chair: K. L. Everts. Vice Chair: N. Kokalis-Burelle. Past Chair: E. N. Rosskoff. K. O. Britton, C. R. Cisar, L. E. Hanson, R. L. Koenig, D. B. Lopes, A. Okoli, S. C. Rabatin, M. Smither-Kopperl, A. B. Thornton, J. M. Windes

Youth Program: Chair: C. H. Walkinshaw. Vice Chair: A. H. Tally. Past Chair: C. A. Jasalavich. A. J. Anderson, C. R. Curtis, K. E. Duncan, J. Esnard, D. W. Kalb, J. M. Krupinsky, R. N. Raid, G. E. Ruhl, J. L. Rytter, P. W. Tooley

\section{Subject Matter}

Bacteriology: Chair: W. F. Mahaffee. Vice Chair: L. S. Pierson. Past Chair: W. Chun. G. A. Beattie, S. B. Beck von Bodman, C. T. Bull, J. C. Chen, D. L. Coplin, R. Frederick, C. H. Liao, F. J. Louws, P. S. McManus, S. A. Miller, S. Q. Pan, A. R. Poplawsky, K. L. Schroeder, G. W. Sundin

Biochemistry, Physiology, and Molecular Biology: Chair: A. F. Bent. Vice Chair: P. A. Gay. Past Chair: A. F. Bent. C. J. Baker, K. R. Davis, M. B. Dickman, R. Hammerschmidt, H. Leung, S. W. Meinhardt, A. B. Thornton, S. Tuzun, G. Upchurch, C. P. Woloshuk, M. N. Zook

Biological Control: Chair: L. L. Kinkel. Vice Chair: V. O. Stockwell. Past Chair: P. J. Cotty. P. M. Brannen, G. L. Brown, D. Gurian-Sherman, C. L. Hartman, D. M. Huber, J. A. Menge, R. A. Owens, J. L. Parke, D. C. Sands, S. F. Shamoun, D. O. TeBeest, R. S. Utkhede

Chemical Control: Chair: D. C. Thompson. Vice Chair: L. E. Zang. Past Chair: P. Lewis. D. T. Beadle, T. B. Brenneman, A. R. Chase, N. Lalancette, P. M. Matherton, T. A. Melton, G. Olaya, L. A. Payan, M. G. Vathakos, H. L. Ypema
Diagnostics: Chair: (to be decided). Vice Chair: (to be decided). Past Chair: E. M. Bentley. J. W. Beale, W. T. Cobb, B. Hudgins, S. T. Koike, S. M. Lerner, B. Rao, G. E. Ruhl, T. S. Schubert, C. A. Smith, T. C. Stebbins, M. A. Williamson

Diseases of Ornamental Plants and Turf Grasses: Chair: D. L. Clement. Vice Chair: (to be decided). Past Chair: R. J. McGovern. M. J. Boehm, D. L. Clement, A. B. Gould, R. K. Jones, M. J. Klopmeyer, R. X. Latin, T. M. Likens, G. W. Moorman, E. B. Nelson, J. Y. Uchida, S. R. Vann, J. E. Watkins

Environmental Quality and Plant Health: Chair: J. A. Glazener. Vice Chair: M. Simini. Past Chair: C. R. Krause. R. Bulluck, P. A. Gay, M. E. Grebus, D. M. Karasevicz, J. Kotcon, M. S. Krause, J. S. Lamboy, M. Skaria, V. L. Smith

Epidemiology: Chair: N. Grunwald. Vice Chair: C. Thomas. Past Chair: H. Scherm. K. L. Bowen, J. A. Duthie, K. M. Emery, N. Evans, C. Hong, G. Hughes, F. W. Nutter, S. Sanogo, A. H. Van Bruggen

Forest Pathology: Chair: D. R. Bergdahl. Vice Chair: K. O. Britton. Past Chair: J. E. Lundquist. D. Bannwart, L. Bernier, S. Cohen, S. A. Enebak, R. C. Hamelin, L. M. Haugen, F. V. Hebard, J. L. Kerrigan, W. H. Ko, W. J. Otrosina, P. C. Spaine, J. A. Walla, R. S. Winder

Genetics: Chair: L. Rosewich. Vice Chair: D. M. Francis. Past Chair: R. A. Dean. W. Chen, S. E. Gold, B. Harveson, S. Kang, F. N. Lee, R. Nelson, T. L. Peever, T. Plyer, J. B. Rasmussen, N. M. Vera Cruz

Host Resistance: Chair: J. L. Starr. Vice Chair: (to be decided). Past Chair: E. A. Milus. X. Chen, R. L. Clark, A. K. Culbreath, A. E. Dorrance, S. M. Lim, J. A. Thies, S. L. Walker, W. L. Wiebe, J. Wilson, J. M. Windes, T. A. Zitter

Integrated Pest Management: Chair: V. Morton. Vice Chair: (to be decided). Past Chair: G. M. Leavitt. E. M. Bauske, R. D. Cartwright, R. E. Ford, B. J. Jacobsen, A. P. Keinath, D. E. Legard, F. J. Louws, B. E. Paulsrud, G. L. Schuster, E. L. Stromberg, D. C. Thompson

Mycology: Chair: P. V. Oudemans. Vice Chair: F. M. Dugan. Past Chair: L. D. Dunkle. D. M. Beyer, M. A. Cubeta, M. O. Garraway, C. A. Jasalavich, H. C. Kistler, W. H. Ko, R. J. Meyer, C. M. Stiles, L. J. Vaillancourt

Mycorrhizae: Chair: S. Hiremath. Vice Chair: (to be decided). Past Chair: D. D. Douds. P. Bonafante, M. Chalot, J. F. Dames, M. Harrison, D. Merhaut, S. R. Shafer

Mycotoxicology: Chair: F. Trail. Vice Chair: G. P. Munkvold. Past Chair: P. J. Cotty. C. W. Bacon, K. F. Cardwell, A. E. Desjardins, J. P. Duvick, M. P. McMullen, D. Wilson, C. P. Woloshuk

Nematology: Chair: L. A. Payan. Vice Chair: (to be decided). Past Chair: R. F. Davis. T. J. Baum, J. O. Becker, P. A. Donald, C. S. Johnson, S. R. Koenning, J. A. LaMondia, D. Neher, T. L. Niblack, R. K. Niles, R. D. Riggs, D. P. Schmitt, E. J. Sikora, B. P. Singh

Pathogen Resistance: Chair: W. D. Koeller

Phyllosphere Microbiology: Chair: P. S. McManus. Vice Chair: H. C. Hoch. Past Chair: J. H. Andrews. D. Aylor, M. Brandl, E. Bustamant, L. J. Giesler, J. C. Hubbard, L. L. Kinkel, H. C. Mellinger, J. Mercier, C. C. Mundt, R. L. Nicholson, V. O. Stockwell

Plant Disease Detection: Chair: J. C. Rupe. Vice Chair: R. R. Martin. Past Chair: D. James. V. DeHerrera, S. N. Jeffers, P. D. Kharbanda, C. A. Levesque, L. Levy, M. G. Tiffany

Plant Disease Losses: Chair: M. H. Royer. Vice Chair: F. W. Nutter, Jr. Past Chair: G. Hughes. S. Chakraborty, J. P. Damicone, J. A. Duthie, T. R. Gottwald, D. Long, Y. Luo, G. L. Tylka, A. Wrather, C. L. Xiao

Postharvest Pathology: Chair: J. A. Bartz. Vice Chair: (to be decided). Past Chair: T. V. Suslow. J. A. Bartz, B. D. Bruton, K. F. Cardwell, W. S. Conway, G. J. Holmes, C.-H. Liao, R. G. McGuire, G. P. Munkvold, D. A. Schisler, P. L. Sholberg, J. L. Smilanick

Seed Pathology: Chair: W. Wiebe. Vice Chair: A. Alvarez. Past Chair: R. L. Forster. G. C. Bergstrom, H. Bouzar, G. P. Hoyos, C. J. Kurowski, J. W. Lorbeer, B. Lovic, C. Thompson, A. Van Buren, R. A. Welliver

Soil Microbiology and Root Diseases: Chair: B. K. Duffy. Vice Chair: K. V. Subbarao. Past Chair: B. Larkin. R. G. Bhat, W. Chen, D. R. Fravel, J. A. Gracia-Garza, J. Graham, R. G. Linderman, R. D. Martyn, Jr., C. S. Rothrock, N. Shishkoff, M. L. Smitther-Kopperl,

Tropical Plant Pathology: Chair: M. J. Davis. Vice Chair: J. Y. Uchida. Past Chair: D. J. Norman. A. Alvarez, S. J. Bagenski, J. J. Cho, J. Comstock, R. F. Lee, A. A. MacNab, N. Martinez-Ochoa, R. A. Naidu, D. J. Norman

Virology: Chair: J. N. Culver. Vice Chair: (to be decided). Past Chair: C. M. Deom. J. K. Brown, J. J. Cho, L. L. Domier, R. C. French, D. T. Gordon, R. Grumet, R. W. Hammond, H. R. Pappu, K. L. Perry, K. M. Scheets, H. B. Scholthof, W. M. Wintermantel, G. C. Wisler, M. Zaitlin

\section{APS Press Committees}

Illustrations of Plant Pathogens and Diseases: Chair: G. L. Schumann. Vice Chair: E. J. Braun. G. J. Holmes, G. W. Hudler, V. Kumar, G. W. Moorman, N. R. O’Neill, J. E. Partridge, A. C. Schilder, G. R. Stanosz, T. J. Volk, J. E. Watkins Phytopathological Classics: Chair: P. D. Peterson. Vice Chair: (to be decided). Past Chair: T. D. Murray. C. L. Campbell, D. W. Fulbright, C. M. Liddell, R. C. Ploetz, K.-B. Scholthof

Standardization of Common Names for Plant Diseases: Chair: B. L. Teviotdale. Vice Chair: M. Putnam. P. F. Bertrand, H. Esteban, C. L. Kohls, M. E. Matheron, G. P. Munkvold, W. T. Nishijima, C. N. Ocamb, D. M. Rizzo, H. E. Waterworth, J. L. W. Woodward, T. A. Zitter 